\title{
Magneto-optical control and spin coupling with non-reciprocal surface waves for nanoscale thermotronics -INVITED
}

\author{
Svend-Age Biehs ${ }^{1, *}$ \\ ${ }^{1}$ Institut für Physik, Carl von Ossietzky Universität, D-26111 Oldenburg, Germany
}

\begin{abstract}
Magneto-optical materials have been proposed as promising candidates for an active control of the directionality of nanoscale heat radiation. Here, we discuss the possibility to rectify nanoscale radiative heat fluxes by means of non-reciprocal surface waves and propose a nanoscale heat flux rectifier or diode which can be controlled actively by means of externally applied fields. We furthermore, reveal the spin coupling mechanism behind the observed heat flux rectification
\end{abstract}

\section{Introduction}

Magneto-optical or more generally non-reciprocal materials have been proposed as promising candidates for an active control of the directionality of nanoscale heat radiation. Unexpected and very interesting effects like the thermal radiative Hall effect [1], persistent currents [2], giant magneto-resistance [3, 4], and circular heat fluxes [5] as well as persistent angular momentum and spin of thermal radiation [5] have been highlighted. We review some of the recent developments in this new direction of magneto-optical thermotronics [6]. In particular, we discuss the possibility to rectify nanoscale radiative heat fluxes by means of nonreciprocal surface waves and propose a nanoscale heat flux rectifier or diode which can be controlled actively by means of externally applied fields [7]. We furthermore, reveal the spin coupling mechanism behind the observed heat flux rectification [8].

\section{Discussion}

Based on fluctuational electrodynamics we derive the general expressions for mean Poynting vector and the heat exchange $[7,8]$ in a general configuration of $N$ nanoparticles in dipolar approximation with temperatures $T_{1}, \ldots, T_{N}$ in an arbitrary environment at temperature $T_{b}$. These general expressions allow us for the special case $N=2$ to evaluate the power exchanged between two InSb nanoparticles and the mean heat flux around the nanoparticle when placing both nanoparticles in close vicinity of an InSb substrate in Voigt configuration as shown in Fig. 1.

Without magnetic field the material properties of the the nanoparticles and the substrate are reciprocal and hence the heat flux in the forward and backward direction turns out to be the same. However, when turning on a magnetic field the material properties become nonreciprocal due to the Lorentz force acting on the electrons in InSb. As a consequence the

\footnotetext{
*e-mail: s.age.biehs@uol.de
} 

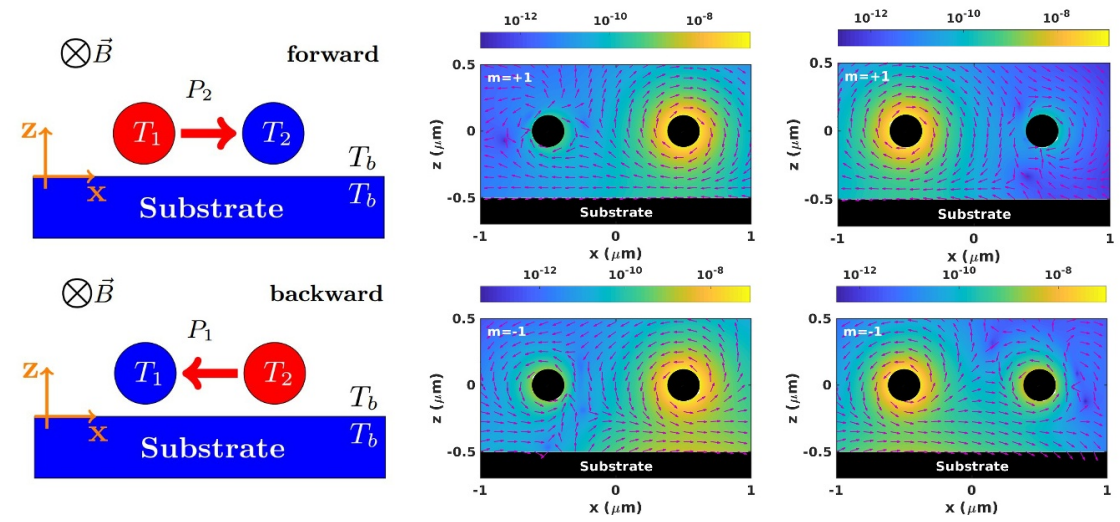

Figure 1. Directional heat flux in forward and backward direction due to the coupling of the $m= \pm 1$ resonances with the spin-momentum locked non-reciprocal surface modes for InSb.

heat flux in forward and backward direction has different values so that the interparticle heat flux becomes rectified. This effect is very strong if the particle resonances can couple to the surface mode resonances. As we will discuss in detail, this coupling is spin selective so that the particle resonance with magnetic quantum number $m=-1(m=+1)$ couples preferably to surface waves travelling in positive (negative) $x$-direction as can be seen in Fig. 1.

This coupling to the non-reciprocal surface modes can be controlled by the amplitude of the external magnetic field and can lead to a very large rectification ratio close to one. We will discuss in detail the dependence on the particle surface distance and the interparticle distance as well as the dependence on the magnetic field amplitude. Since the heat emitted by the hot particle will not only heat up the cold particle but also the surface itself there is a competition between the interparticle and the particle-surface heat flux. Therefore we study the heating dynamics in forward and backward direction to quantify the heating efficiencies.

\section{References}

[1] P. Ben-Abdallah, "Photon Thermal Hall Effect," Phys. Rev. Lett. 116, 084301, (2016).

[2] L. Zhu and S. Fan, "Persistent Directional Current at Equilibrium in Nonreciprocal Many-Body Near Field Electromagnetic Heat Transfer," Phys. Rev. Lett. 117, 134303 (2016).

[3] I. Latella and P. Ben-Abdallah, "Giant Thermal Magnetoresistance in Plasmonic Structures,” Phys. Rev. Lett. 118, 173902, (2017).

[4] R. M. Abraham Ekeroth, P. Ben-Abdallah, J.C. Cuevas, and A. Garcia Martin, "Anisotropic Thermal Magnetoresistance for an Active Control of Radiative Heat Transfer," ACS Photonics 5, 705 (2017).

[5] A. Ott, P. Ben-Abdallah, and S.-A. Biehs, "Circular heat and momentum flux radiated by magneto-optical nanoparticles,” Phys. Rev. B 97, 205414 (2018).

[6] A. Ott, R. Messina, P. Ben-Abdallah, S.-A. Biehs, "Magnetothermoplasmonics: from theory to applications," J. Photon. Energy 9, 032711 (2019).

[7] A. Ott, R. Messina, P. Ben-Abdallah, S.-A. Biehs, "Radiative thermal diode driven by nonreciprocal surface waves," Appl. Phys. Lett. 114, 163105 (2019).

[8] A. Ott and S.-A. Biehs, "Thermal rectification and spin-spin coupling of non-reciprocal localized and surface modes," Phys. Rev. B 101, 155428 (2020). 